\title{
Bupropion Induced Serotonin Syndrome: A Case Report
}

\author{
Elizabeth L. Thorpe • Anthony F. Pizon • \\ Michael J. Lynch • Jessica Boyer
}

Published online: 18 March 2010

(C) American College of Medical Toxicology 2010

\begin{abstract}
Although there are no documented cases of serotonin syndrome (SS) following bupropion ingestion alone in the literature, the ability of bupropion to potentiate serotonin levels and lead to SS is known. A 15 -year-old boy was found at home hallucinating. He then developed tonicclonic activity. Upon arrival in the emergency department, he was confused and restless. On exam, he had tachycardia, hypertension, dilated pupils and dry oral mucosa, normal tone and reflexes in his arms, but rigidity and +4 reflexes in his legs with sustained clonus at his ankles. He was admitted and treated with intravenous fluids and lorazepam for his agitation. A urine drug screen (via gas chromatography/mass spectrometry) was positive only for naproxen and bupropion.
\end{abstract}

Funding Sources: None

Accepted as a poster presentation for the North American Congress of Clinical Toxicology Annual Meeting 2009, San Antonio, TX, USA.

E. L. Thorpe · A. F. Pizon • M. J. Lynch

Department of Pediatrics, Division of Emergency Medicine, University of Pittsburgh School of Medicine,

Pittsburgh, PA, USA

\section{A. F. Pizon $(\varangle) \cdot$ M. J. Lynch}

Department of Emergency Medicine, Division of Medical

Toxicology, University of Pittsburgh School of Medicine,

Presbyterian, 200 Lothrop Street, Suite DL-45,

Pittsburgh, PA 15213, USA

e-mail: pizonaf@upmc.edu

\section{J. Boyer}

Department of Emergency Medicine, UPMC St. Clair, Pittsburgh, PA, USA
Serum bupropion and hydroxybupropion levels drawn 17 $\mathrm{h}$ after his reported ingestion were 280 (therapeutic range $50-100$ ) and $3,100 \mathrm{ng} / \mathrm{mL}$ (therapeutic range $<485$ ), respectively. Within $24 \mathrm{~h}$ of his admission, the patient was awake with normal vital signs and neurologic exam. To our knowledge, there are only three reported cases demonstrating SS in conjunction with bupropion toxicity; however, none of these were secondary to bupropion alone.

Keywords Bupropion $\cdot$ Serotonin syndrome $\cdot$ Pediatrics

\section{Introduction}

Serotonin syndrome (SS) is characterized by the triad of altered mental status, autonomic dysfunction, and neuromuscular abnormalities $[1,2]$. It is classically associated with supratherapeutic doses of a single selective serotonin reuptake inhibitor (SSRI) or the combination of two or more serotonergic agents. However, many medications, including monoamine oxidase inhibitors, tricyclic antidepressants, opiate analgesics, weight-reduction agents, antiretroviral agents, and antibiotics have been associated with SS [1-6]. Additionally, SS has been precipitated by a single therapeutic dose of an SSRI and by the addition of drugs that inhibit CYP450 isoenzymes [5, 7, 8]. Recently, bupropion has been reported as a potential causative agent in serotonin syndrome [9-11].

Bupropion is an antidepressant and smoking cessation aid with the potential for toxic effects in overdose that include seizures, tachycardia, hypertension, and agitation 
[12-14]. It selectively inhibits neuronal reuptake of dopamine and norepinephrine and may have indirect effects at serotonergic receptors [15-18]. To date, bupropion has only been reported as a causative agent in serotonin syndrome in conjunction with other medications $[9,10]$. We report a case of serotonin syndrome caused by bupropion ingestion alone.

\section{Case}

A previously healthy 15 year-old Caucasian boy with a history of depression, anxiety, and attention deficit hyperactivity disorder, who was being treated only with bupropion, woke his grandparents in the middle of the night with incoherent shouting. He reported seeing people who were not present and then developed $5 \mathrm{~min}$ of generalized tonic-clonic movements without incontinence or eye deviation. Emergency Medical Services (EMS) were called.

When EMS arrived, the patient was incoherent and agitated. En route to the Emergency Department (ED), he had a second episode of tonic-clonic movements that resolved spontaneously. Upon arrival in the ED, he was confused and restless. Vitals signs included a heart rate of 170 beats per minute, respiratory rate of 24 breaths per minute, blood pressure of $170 / 130 \mathrm{mmHg}$, temperature of $37.1^{\circ} \mathrm{C}$, and oxygen saturation of $98 \%$ on room air. The patient had been healthy and acting normally prior to the onset of these symptoms. His only prescribed medication was extended release bupropion, $300 \mathrm{mg}$ daily administered orally. Initial physical examination documented by the ED physician revealed dilated but reactive pupils, normal conjunctiva, dry oral mucosa, tachycardia, flushed and dry skin, rigidity in his legs, and +4 reflexes in his ankles. The remainder of his examination was unremarkable. He was given $2 \mathrm{~L}$ of intravenous (IV) fluids and a total of $8 \mathrm{mg}$ of lorazepam administered intravenously, which decreased his agitation. Initial labs revealed unremarkable electrolytes, blood urea nitrogen, and creatinine. Total creatine phosphokinase and white blood count were elevated at $991 \mathrm{IU} / \mathrm{L}$ (reference range 0-200 IU/L) and $19,900 / \mathrm{mm}^{3}$ (reference range $\left.4,500-11,000 / \mathrm{mm}^{3}\right)$, respectively. Serum ethanol, salicylate, and acetaminophen levels were undetectable. Electrocardiogram demonstrated a heart rate of 170 beats per minute, QRS of $100 \mathrm{~ms}$, and QTc of $434 \mathrm{~ms}$. Unenhanced computed tomography of the head was normal, and the patient was transferred to the regional toxicology treatment center for further evaluation and treatment.

The patient remained delirious and agitated upon arrival at our facility. Vital signs were unchanged. Repeat physical examination showed dry mucous membranes and mydriasis.
He was not diaphoretic. Neurologic examination was notable for hyperreflexia and clonus in the lower extremities, while upper extremity reflexes remained normal. Additionally, he was extremely rigid in his lower extremities, but his upper extremities had normal tone. He was given $2 \mathrm{mg}$ of physostigmine administered intravenously without effect. Comprehensive urine drug screen by gas chromatography/mass spectrometry was positive only for naproxen and bupropion. The patient was admitted to an inpatient unit and treated supportively with IV fluids and additional lorazepam.

Serum bupropion and hydroxybupropion levels drawn $17 \mathrm{~h}$ after his reported ingestion were $280 \mathrm{ng} / \mathrm{mL}$ (therapeutic range $50-100 \mathrm{ng} / \mathrm{mL}$ ) and $3,100 \mathrm{ng} / \mathrm{mL}$ (therapeutic range $<48 \mathrm{ng} / \mathrm{mL}$ ), respectively. Within $24 \mathrm{~h}$ of admission, the patient was awake with normal vital signs and neurologic examination (his symptoms resolved less than $36 \mathrm{~h}$ after the reported time of ingestion). He admitted to taking 10 tablets of his $300 \mathrm{mg}$ sustained-release bupropion. The patient was transferred to an inpatient psychiatric facility following symptom resolution.

\section{Discussion}

A Medline search of the English language literature demonstrated only three case reports of serotonin syndrome in conjunction with bupropion ingestion. None of these were secondary to bupropion alone or included pediatric patients [9-11]. Munhoz reported a case of a 62-year-old woman who developed SS from a combination of bupropion, sertraline, piracetam, and venlafaxine [9]. She had subtle signs of SS after 3 weeks of bupropion and sertraline alone and then acutely worsened after the addition of venlafaxine and piracetam. Szaklay and Strauss report a case of possible SS in a patient undergoing oral surgery who had taken his regular dose of bupropion plus a onetime dose of dextromethorphan [10]. Dvir and Smallwood describe a 53-year-old woman who was on fluoxetine, and olanzapine daily, and developed SS with the addition of bupropion to her regimen [11].

The toxicity of bupropion in children and adolescents has been reported in multiple cases, but none have described serotonin syndrome. For example, Ayers and Tobias describe an adolescent with tachycardia, slurred speech, dry skin, ataxia, and seizures but no hyperreflexia following intentional ingestion of 15 to $30100-\mathrm{mg}$ tablets of bupropion [19]. Givens and Gabrysch reported an ingestion of $2 \mathrm{~g}$ of bupropion by a 3-year-old child that resulted in seizures and profound hemodynamic instability in conjunction with complications from whole bowel irrigation [20]. Finally, Spiller and Schaeffer report seizures, hallucinations, ataxia, mydriasis, and moist mucous 
membranes in a 7-year-old boy following ingestion of $1,050 \mathrm{mg}$ of bupropion [21]. None of the above reports describe the classic findings of lower extremity hyperreflexia and clonus that are seen in SS. Some cases fail to mention reflexes at all, and therefore, the presence of SS in these cases is difficult to confirm.

The main pathophysiologic mechanism for SS appears to be excessive 5- hydroxytryptophan subtype (5HT1a and 5HT2a) stimulation $[1,2]$. The serotonergic effects of bupropion have been debated. In humans, some studies have concluded that bupropion has no serotonergic activity $[15,22]$. However, studies in animals have shown that bupropion may have some serotonergic activity [17, 18, 23]. For instance, Piacentini et al. found a comparable increase in the levels of serotonin, norepinephrine, and dopamine in the hippocampus of rats after administration of bupropion. Others have postulated that bupropion may have indirect serotonergic activity through its up regulation of vesicular monoamine transporter-2, the transporter responsible for pumping dopamine, norepinephrine, and serotonin from the cytosol into presynaptic vesicles [24].

The differential diagnosis of serotonin syndrome includes anticholinergic poisoning, malignant hyperthermia (MH), and neuroleptic malignant syndrome (NMS), and CNS infection, each of which can be distinguished from SS by history and physical exam in conjunction with the appropriate laboratory evaluation. While our patient had some stigmata of anticholinergic poisoning including mydriasis, agitated delirium, hot and dry skin, and urinary retention, he had no response to physostigmine or exposure to anticholinergic medications. His lack of ingestion of anticholingeric medications is supported by urine evaluation by GC/MS, making anticholinergic toxicity very unlikely. His dry oral mucosa and skin were interpreted as signs of dehydration and not anticholinergic findings. The patient had no exposure to neuroleptic medications, inhaled anesthetics, or succinylcholine which would be required to diagnose NMS or MH. On exam, he was not febrile, and his neck was supple, making a CNS infection less likely. Certainly, rapid improvement in his symptoms without the need for antibiotics makes a life-threatening infection unlikely. The patient's symptoms of tachycardia, mydriasis, urinary retention, and altered mental status are all consistent with previously reported cases of serotonin syndrome [25]. Many of these symptoms are also similar to bupropion toxicity without SS. However, the unique finding of lower extremity hyperreflexia and clonus with normal upper extremity reflexes is characteristic of SS and supports this diagnosis in the patient presented. While the patient's clinical presentation fits the diagnostic criteria for SS described by Sternbach and Radomski, he did not develop hyperthermia, a classically reported finding in SS [25, 26]. A review of 62 cases of SS reported to medical journals found the incidence of hyperthermia to be only $37.5 \%$ and diaphoresis to be $33.3 \%$ [25]. Therefore, the lack of hyperthermia and diaphoresis does not preclude a diagnosis of SS. Adequate sedation with benzodiazepines may have prevented hyperthermia caused by ongoing muscle hyperactivity, and his dehydration may have contributed to the lack of sweating.

There were two potential limitations to this study. First, cyproheptadine, a serotonin antagonist that has been used anecdotally in cases of SS, was not used in this case [27]. While there is a favorable risk-benefit ratio, there is a lack of randomized clinical data to support its use. Second, it is possible that a drug other than bupropion could have caused these symptoms. This is unlikely given that the comprehensive urine drug screen by gas chromatography/mass spectrometry done at our institution screens for most, if not all, SSRIs.

In conclusion, we believe this to be the first case report of bupropion toxicity alone causing serotonin syndrome.

\section{References}

1. Martin TG (1996) Serotonin syndrome. Ann Emerg Med 28:520 526

2. Boyer EW, Shannon M (2005) The serotonin syndrome. NEJM 352:1112-1120

3. Giese SY, Nebrosky R (2001) Serotonin syndrome: potential consequences of Meridia combined with demorol or fentanyl. Plast Reconst Surg 107:293-294

4. DeSilva KE, Le Flore DB, Marston BJ et al (2001) Serotonin syndrome in HIV infected individuals receiving antiretroviral therapy and fluoxetine. AIDS 15:1281-1285

5. Lee DO, Lee CD (1999) Serotonin syndrome in a child associated with erythromycin and sertraline. Pharmacotherapy 19:894-896

6. Lane R, Baldwin D (1997) Selective serotonin reuptake inbitiborinduced serotonin syndrome: review. J Clin Psychopharmacol 17:208-221

7. Gill M, LoVecchio F, Selden B (1999) Serotonin syndrome in a child after a single dose of fluvoxamine. Ann Emerg Med 33:457459

8. Mitchell PB (1997) Drug interactions of clinical significance with selective serotonin reuptake inhibitors. Drug Saf 17:390-406

9. Munhoz RP (2004) Serotonin syndrome induced by a combination of bupropion and SSRI's. Clin Neuropharmacol 27:219-222

10. Szakaly D, Strauss R (2008) Serotonin syndrome in the oral and maxilofacial surgery office: a review of the literature and report of a case. J Oral Maxillofac Surg 66:1949-195

11. Dvir Y, Smallwood P (2008) Serotonin syndrome: a complex but easily avoidable condition. Gen Hosp Psych 30:284-287

12. Balit CR, Lynch CN, Isbister GK (2003) Bupropion poisoning: a case series. Med J Aust 178(2):61-63

13. Belson MG, Kelley TR (2002) Bupropion exposures: clinical manifestations and medical outcome. J Emer Med 23:223-230

14. Horst WD, Preskorn SH (1998) Mechanisms of action and clinical characteristics of three atypical antidepressants: venlafaxine, nefazodone, buproion. J Affect Disord 51:237-254

15. Ascher JA, Cole JO et al (1995) Bupropion: a review of its mechanism of antidepressant activity. J Clin Psychiatry 56 (9):395-401 
16. El Mansari M, Ghanbari R et al (2008) Sustained administration of bupropion alters the neuronal activity of serotonin, norepinephrine but not dopamine neurons in the rat brain. Neuropharmacology 55 (7):1191-8

17. Cooper BR, Wang CM et al (1994) Evidence that the acute behavioral and electrophysiologic effects of bupropion are mediated by a noradrenergic mechanism. Neuropsychopharmacology 11:133141

18. Dong J, Blier P (2001) Modification of norepinephrine and serotonin, but not dopamine, neuron firing by sustained bupropion treatment. Psychopharmacology 155:52-57

19. Ayers S, Tobias JD (2001) Bupropion overdose in an adolescent. Pediatr Emerg Care 17(2):104-106

20. Givens ML, Gabrysch J (2007) Cardiotoxicity associated with accidental bupropion ingestion in a child. Pediatr Emerg Care 23 (4):234-237

21. Spiller HA, Schaeffer SE (2008) Multiple seizures after bupropion overdose in a small child. Pediatr Emerg Care 24(7):474-475
22. Stahl SM, Pradko JF et al (2004) A review of the neuropharmacology of bupropion, a dual norepinephrine and dopamine reuptake inhibitor. Prim Care Companion J Clin Psychiatry 6 (4):159-166

23. Piacentini MF, Clinckers R, Meeusen R et al (2003) Effect of bupropion on hippocampal neurotransmitters and on peripheral hormonal concentrations in the rat. J Applied Physiol 95:652-656

24. Foley KF, DeSanty KP, Kast RE (2006) Bupropion: pharmacology and therapeutic applications. Expert Rev Neurother 6(9):1246-1265

25. Radomski JW, Dursun SM, Reveley MA, Kutcher SP (2000) An exploratory approach to the serotonin syndrome: an update of clinical phenomenology and revised diagnostic criteria. Med Hypotheses 55(3):218-24

26. Sternbach H (1991) The serotonin syndrome. Am J Psychiatry 148:705-713

27. Horowitz BZ, Mullins ME (1999) Cyproheptadine for serotonin syndrome in an accidental pediatric sertraline ingestion. Pediatr Emerg Care 15:325-327 